\title{
Synthesis and Properties of Macrocyclic Complexes Ruthenium Azerbaijan State University of Oil and Industry
}

\author{
Mikayilova Mexriban Rahil, Mustafayeva Rena Eldar \\ Scientific-Research Laboratory «New Chemical Materials and Technologies», "Azerbaijan State University of Oil and Industry", Baku, \\ Republic of Azerbaijan
}

Email address:

rena-babaeva0@rambler.ru (M. R. Eldar)

\section{To cite this article:}

Mikayilova Mexriban Rahil, Mustafayeva Rena Eldar. Synthesis and Properties of Macrocyclic Complexes Ruthenium Azerbaijan State University of Oil and Industry. American Journal of Heterocyclic Chemistry. Vol. 4, No. 1, 2018, pp. 22-25.

doi: 10.11648/j.ajhc.20180401.12

Received: October 18, 2017; Accepted: November 23, 2017; Published: January 29, 2018

\begin{abstract}
Synthetic methods have been developed and Ru (IV) complex compounds with five types of donor ligands have been prepared: phenanthroline, nicotinic acid amide, imidazole, benzimidazole and their derivatives, and phthalocyanine. New method for the synthesis of rhenium and ruthenium phthalocyanines is proposed, which is distinguished by its availability and high yields. It is established that because of synthesis only one form of ruthenium phthalocyanine is formed, which can be attributed to the PcRuO structure based on physical and chemical studies.
\end{abstract}

Keywords: Complex Compounds, Ruthenium, Nicotinic Acid Amide, Donor Ligands, Palladium

\section{Introduction}

Ruthenium is the first chemical element discovered by the Russian chemist Karl Karlovac Klaus. Ruthenium, the representative of platinum metals, was discovered by the latter among them. A chemical element is named after Russia (the Latin name of Russia is Ruthenia) [1].

Ruthenium has many valuable and interesting properties. For many mechanical, electrical and chemical characteristics, it can compete with many metals and even with platinum and gold. However, unlike these metals, ruthenium is very fragile, and therefore it is not possible to make any products from it yet. Apparently, the brittleness and intractability of ruthenium by mechanical treatment are due to the insufficient purity of the samples subjected to the tests.

\section{Experimental}

The physical properties of this metal depend very much on the method of production, and no one has yet been able to isolate ruthenium of high purity. Attempts to obtain pure ruthenium by sintering in briquettes, zone melting and other methods did not lead to positive results. For this reason, technically important characteristics such as tensile strength and elongation at break have not yet been accurately determined. Only recently, the melting point of ruthenium is accurately estimated at $2,250^{\circ} \mathrm{C}$, and its boiling point lies somewhere around $4,900^{\circ} \mathrm{C}$. Metal ruthenium very actively sorbs hydrogen. Usually the standard of the hydrogen sorbent is palladium, the cubic centimeter of which absorbs $940 \mathrm{~cm}^{3}$ of hydrogen. The absorption capacity of ruthenium is higher. It sorbs 1,500 volumes of hydrogen [2].

Compact metallic ruthenium is not soluble in alkalis, acids and even in boiling aqua Regia, but partially soluble in nitric acid with the addition of strong oxidizing agents - per chlorates or bromates.

Ruthenium can be dissolved in alkaline medium by hypochlorite's or in acid medium by electrochemical method.

When heated in air, ruthenium begins to partially oxidize. The maximum oxidation rate is observed at $800^{\circ} \mathrm{C}$. Up to a temperature of $1,000^{\circ} \mathrm{C}$, ruthenium is always oxidized only to $\mathrm{RuO}_{2}$, but if it is heated to $1,200^{\circ} \mathrm{C}$ and higher, it begins to convert to volatile $\mathrm{RuO}_{4}$ tetra oxide, exhibiting a higher valence of +8 .

$\mathrm{RuO}_{4}$ is a very interesting compound. Under normal conditions, these are golden yellow needle-shaped crystals that already melt at $25^{\circ} \mathrm{C}$, turning into a brown-orange liquid with a specific odor similar to that of ozone. When touched with the slightest trace of most organic substances, ruthenium tetroxide momentarily explodes. At the same time, it 
dissolves well in chloroform and carbon tetrachloride. $\mathrm{RuO}_{4}$ is poisonous: with prolonged inhalation of its vapors, the person begins to feel dizzy, there are attacks of vomiting and suffocation. Some chemists who worked with ruthenium tetroxide developed eczema.

Thus, the synthesized hybridization on of polymerimmobilized $\mathrm{Ru}$ nanoparticles show high activity in cyclohexene hybridization reaction and keeps its activity during repeated cycles. The catalytic properties of the Nano composites depend on the conditions of their preparation and it affects the size of the formed Ru nanoparticles.

The ability of ruthenium to form tetroxide played an important role in the chemistry of this element. By transferring to volatile $\mathrm{RuO}_{4}$, it is possible to separate ruthenium from other noble and base metals and, after its recovery, obtain the most pure ruthenium. In the same way, ruthenium impurities are removed from rhodium, iridium and platinum [3].

The struggle against radioactive ruthenium is paid much attention to by physicists, chemists, technologists and especially radio chemists of many countries. At the First and Second International Conferences on the Peaceful Uses of Atomic Energy in Geneva, several reports were devoted to this problem [4].

Ruthenium is the only platinum metal found in living organisms (according to some sources, it is also platinum). It concentrates mainly in the muscle tissue. The highest ruthenium oxide is extremely toxic and, being a strong oxidant, can cause fire of fire hazardous substances.

Ruthenium is a rare and very scattered element. In industry heterogeneous catalysis uses metallic ruthenium and its alloys. Ruthenium is used in the preparation of the most effective catalysts. The present study was carried out in the field of synthetic and structural chemistry of ruthenium complexes with organic nitrogen-containing compounds.

Using ruthenium $\mathrm{Ru}_{3}(\mathrm{CO})_{12}$ as a starting compound and reacting with a phthalocyanine ligand in benzonitrile for 1.5 hours followed by extraction with pyridine, it was possible to obtain in a small yield a ruthenium complex with phthalocyanine containing axially coordinated $\mathrm{CO}$ molecules and pyrolysine, $\mathrm{Ru} \mathrm{Pc}(\mathrm{CO})$.

Alkyl-substituted ruthenium phthalocyanine's were synthesized from free phthalocyanine with higher yields. $\left(\mathrm{C}_{5} \mathrm{H}_{11} \mathrm{O}\right) \mathrm{SP}_{2} \mathrm{H}_{2}$ and dissolved in boiling 2-ethoxyethanol, a solution of $\mathrm{RuCl}_{3} \times 3 \mathrm{H}_{2} \mathrm{O}$ in the same solvent (molar ratio of starting reactants 1:2) was added and the mixture was boiled for 24 hours.

The cooled solution was poured into $\mathrm{MeOH}: \mathrm{H}_{2} \mathrm{O}$ (3:1), filtered and dried. The reaction product was then purified by chromatography on neutral alumina $\mathrm{Al}_{2} \mathrm{O}_{3}$ (eluentchloroform). However, this method failed to obtain a complex without axial ligands: the IR spectrum of the compound synthesized in this way contains a characteristic band at 1900 $\mathrm{cm}-1$, characterizing the valence vibrations of - (CO). In the 13C-NMR spectrum of this complex, a signal is observed in the region of $181.06 \mathrm{ppm}$, which also confirms the presence of a $\mathrm{CO}$ molecule in the complex. The yield $\left(\mathrm{C}_{5} \mathrm{H}_{11} \mathrm{O}\right)_{8} \mathrm{PcRu}$ (CO) was $30 \%$, for (2-Et-hexO) ${ }_{8} \mathrm{PcRu}(\mathrm{CO}) 25 \%$.
This, on the methods of synthesis and the composition of substituted and unsubstituted ruthenium phthalocyanines, it can be concluded that the final product of the reaction, regardless of the method of synthesis, almost always contains in its composition a carbonyl group of $\mathrm{CO}$ as an axial ligand.

It was found that the production of pure ruthenium phthalocyanine, which does not contain any axially coordinated molecules, is possible with the thermal decomposition of complexes of the composition PcRu (CO) $\mathrm{L}$ or $\mathrm{PcRuL}_{2}$. Therefore, many works are devoted to the synthesis and study of ruthenium complexes with phthalocyanines and various axial ligands.

One of the most common methods for obtaining such complexes is the interaction of the original "PcRu" obtained by template synthesis with the corresponding ligand L. This, in the article, compounds containing as molecules of DMSO and DMFA as axial ligands, except carbonyl group $\mathrm{CO}$, were obtained. The original unsubstituted ruthenium phthalocyanine "RcRu" was boiled in an appropriate solvent for 3 and 5 hours. The reaction mass was then chromatographed on neutral alumina with $\mathrm{Al}_{2} \mathrm{O}_{3}$ hexane to remove the excess solvent, and then using the complexes PcRu (DMFA) (CO) '(20\% yield) and PcRu (DMSO) (CO) (Yield 40\%).

Investigating the interaction between "PcRu" and 2,3,5, btetramethyl-1,4-diisocyanobenzene ( $\left.\mathrm{Me}_{4} \mathrm{dib}\right)$ or 2,3,5,6tetrachloro-1,4-diisocyanobenzene $\left(\mathrm{Cl}_{4} \mathrm{dib}\right)$, depending on the molar ratio Starting reagents and synthesis conditions, monomeric or polymeric ruthenium complexes are formed as reaction products (Figure 1).

Reaction of the composition PcRu $\left(\mathrm{X}_{4} \mathrm{clib}\right)_{2}(\mathrm{~V})$, where (X $=\mathrm{Me}, \mathrm{C} 1)$, was $62 \%$. Polymer composition complexes were prepared by reacting identical molar amounts of the starting reagents by heating at $\mathrm{t}=25^{\circ} \mathrm{C}$ for $24-28$ hours in acetone. The purification was performed by extraction with acetone for 48 hours. The yield of [PcRu (X)] n (VI) (n- 10, X = $\mathrm{Me}_{4} \mathrm{dib}, \mathrm{Cl}_{4} \mathrm{dib}, \mathrm{Me}$ dib, $\mathrm{Me}_{2} \mathrm{dib}, \mathrm{Bu}_{2} \mathrm{dib},\left(\mathrm{C}_{8} \mathrm{H}_{17}\right)_{2} \mathrm{dib}$, $\left(\mathrm{C}_{10} \mathrm{H}_{21}\right)_{2}$ dib was $95-100 \%$.
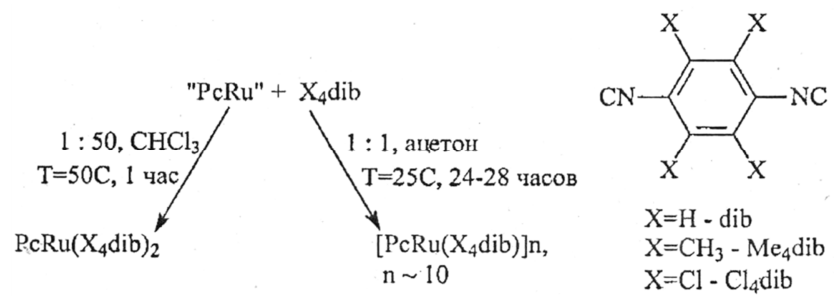

Figure 1. Monomeric or polymeric ruthenium complexes.

In a similar way, And polymeric composition with unsubstituted 1,4-diisocyanobenzene (dib) in 85\% yield and $95 \%$, respectively. To synthesize the $\mathrm{Pc}-\mathrm{Fe}$ and $\mathrm{Pc}-\mathrm{Ru}$ complexes, $\mathrm{NH}_{4} \mathrm{RuO}_{4}, \quad \mathrm{RuCl}_{3} \quad 3 \mathrm{H}_{2} \mathrm{O}$ and 4-tertbutylphthalodinitrile were chosen as the starting compounds. All solvents and starting materials were purified immediately prior to use according to standard procedures. [9, 10]

In the IR spectrum (figure 2.) of the complexes there is a band 940-945 cm-1, which according to the data of [5] corresponds to the relation $\mathrm{Ru}=0$. The absence of any bands 
in the interval $3100-3700 \mathrm{~cm}-1$ indicates that the molecules do not contain $\mathrm{OH}$ groups either in the ligand or in the metal atom.

The mass spectra contain a peak of a molecular ion with a mass of 939, which corresponds to the formula $4-(\mathrm{t}-\mathrm{Bu}){ }_{4} \mathrm{Pc}$ $\mathrm{ReO}$ and a peak molecular ion with a mass of 829 , which corresponds to the formula 4- $(\mathrm{t}-\mathrm{Bu}){ }_{4} \mathrm{Pc} \mathrm{RuO}$. Mass spectrometry data support the assumption that the compounds obtained have the structure of PcRuO. In these complexes, the degree of oxidation of rhenium is five, and ruthenium is equal to four (Figure 3 ).

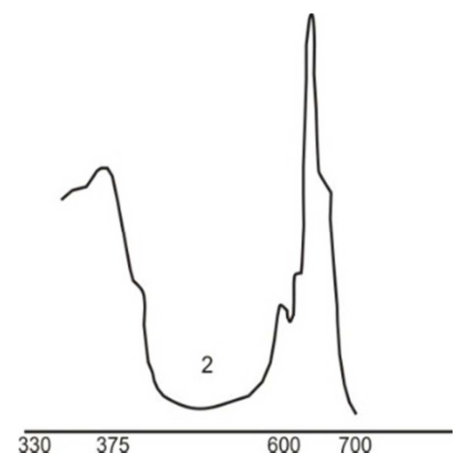

Figure 2. Electronic absorption spectra of phthalocyanines Ruthenium in benzoyl.

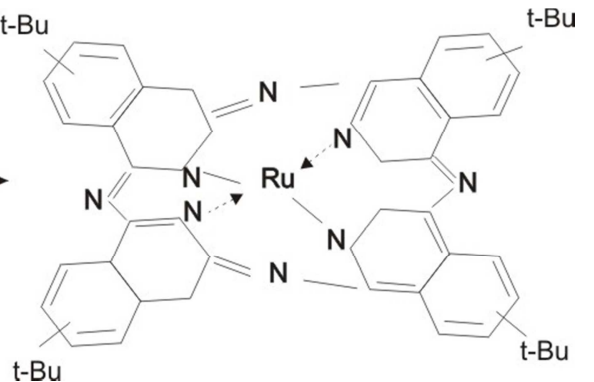

Figure 3. The compounds obtained have the structure of PcRuO.

New methods for the synthesis of rhenium and ruthenium phthalocyanines are proposed. These methods are characterized by availability and high yields. It was found that because of the synthesis only one form of phthalocyanines PcRe and PcRu is formed allowed to attribute to these forms the structure of PcReO and PcRuO (Figure 4) [6].

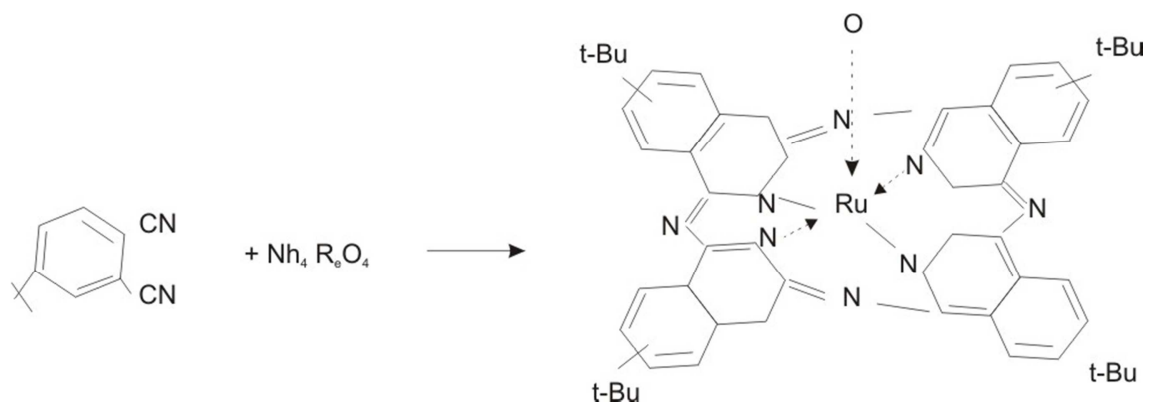

Figure 4. These forms the structure of PcReO and PcRuO.

Complex compounds can be successfully used for the diagnosis and treatment of cancer of various human organs. In this case, the radiotherapeutic preparation can be addressed directly to the patient's diseased organ by means of specific molecular vectors, i. e. In the form of coordination compounds with a certain type of ligands and a certain structure. Active research in this field is carried out in many laboratories of the world: in the USA (Prof. J. Zubieta), in Canada (Prof. ALBoshan), in Germany (dr. H. Shpis, Prof. Herrmann), in Italy (Prof. S. Middolini), in England (Prof. J. R. Dilvars).

\section{Results and Discussion}

The resulting hybrid nanocomposites have a sufficiently developed surface and porous structure, which ensures the availability of active catalyst centers for reagents and their high activity in the catalytic reactions under study.

It was found that the investigated nanocomposites retain the catalytic activity and when repeated cycles of reaction are carried out on them, and the fact that they are in the immobilized form makes it possible to easily separate them from the reaction medium and to use them repeatedly, on the other hand, to study different physic-chemical methods formed on the catalyst intermediates.

Another main area of ruthenium consumption is the production of catalysts for the petrochemical industry, as well as the production of exhaust gas filters for passenger cars. The catalysts promoted by these metals are widely used in various technological processes.

\section{Conclusions}

Synthetic methods have been developed and Ru (IV) complex compounds with five types of donor ligands have been prepared: phenanthroline, nicotinic acid amide, imidazole, benzimidazole and their derivatives, and phthalocyanine. [7]. 
New method for the synthesis of rhenium and ruthenium phthalocyanines is proposed, which is distinguished by its availability and high yields. It is established that because of synthesis only one form of ruthenium phthalocyanine is formed, which can be attributed to the PcRuO structure on the basis of physical and chemical studies. [8].

\section{References}

[1] Raevskaya MV: Physicochemistry of ruthenium and its alloys / MV Raevskaya // Chemistry of ruthenium, rhodium, palladium, osmium, iridium, platinum, per. With the English. M.: 1979.-P. 138-140.

[2] Brockhaus F. A. Encyclopedic Dictionary Brockhaus and I. A. Efron / F. A. Brockhaus, I. A. Efron. - S.-Pb.: Brockhaus Efron, 1890-1907.-347 p.

[3] Prokhorov A. M. Physical encyclopedia in 5 volumes / A. M. Prokhorov, Moscow: Soviet Encyclopedia. Editor-in-chief A. M. Prokhorov. - Volume 3, p. 317-319.

[4] Sokolov I. V. Popular library of chemical elements / I. V. Petryanov-Sokolov // Moscow: Nauka Publishers, 1963. pp. 83-86.
[5] M. Cavazzini, S. Quici, C. Scalera, F. Puntoriero, G. La Ganga, S. Campagna. Synthesis, characterization, absorption spectra, and luminescence properties of multinuclear species made of $\mathrm{Ru}$ (II) and Ir (III) chromophores. // Inorg. Chem.2009.-V. 48.-P. 8578-8592.

[6] R. Lalrempuia, M. R. Kollipara. Rectivity studies of $\eta^{6}$-arene ruthenium (II) dimers with polypyridyl ligands: isolation of mono, binuclear $p$ - cymene ruthenium (II) complexes and bisterpyridine ruthenium (II) complexes. //Polyhedron-2003.V. 22.-P. 3155-316.

[7] C. Mikel, P. G. Potvin. Synthesis and enhanced photosensitization ability of a 4-carboxy-2, 2':6', 2"- terpyridine complex of ruthenium (II)// Polyhedron-2002.-V. 21.-P. 49-54.

[8] J. Mola, E. Mas-Marza, X. Sala, I. Romero, M. Rodriguez, C. Vinas, T. Parella, A. Llobet. Ru-Hbpp-based water-oxidation catalysts anchored on conducting solid supports. // Angew. Chem. Int. Ed.-2008.-V. 47.-P. 5830-5832.

[9] M. Agaguseynova, N. E. Jabbarova Coordination compounds of transition metals in catalysis.// Chem. Soc. Commun. Baku2006 -p. 129-135.

[10] M. Agaguseynova, N. E. Jabbarova Complexes of transition metals with molecular oxygen.// Chem. Soc. Commun. Baku2012 -p. 96-111. 